\title{
DISPERSAL CAN SHARPEN PARAPATRIC BOUNDARIES ON A SPATIALLY VARYING ENVIRONMENT
}

\author{
Gisela García-Ramos, ${ }^{1,5}$ Faustino Sánchez-Garduño, ${ }^{2}$ And Philip K. Maini ${ }^{3,4}$ \\ ${ }^{1}$ Department of Zoology, University of Texas, Austin, Texas 78712 USA \\ ${ }^{2}$ Department of Mathematics, Science Faculty, Universidad Nacional Autónoma de México, \\ Circuito Exterior, Ciudad Universitaria, México, 04510 D.F., México \\ ${ }^{3}$ Centre for Mathematical Biology, Mathematical Institute, University of Oxford, \\ 24-29 St. Giles', Oxford OX1 3LB, UK
}

\begin{abstract}
Parapatry describes a geographic pattern in which the ranges of two species have separate but contiguous distributions without any physical barriers between them. We present results from a study of ecological mechanisms to explain parapatry in closely related species. These include competition, spatially varying performances, and dispersal that depends on the densities of both species. We use a model consisting of two coupled nonlinear reaction-diffusion equations with density-dependent diffusion terms and space-dependent Lotka-Volterra-like competing interaction terms. The model is analyzed by using a mixture of phase-plane analysis and numerical simulations.

Results show that competition and dispersal can lead to completely segregated species ranges. Spatial variation favored and provided stability to parapatric distribution. Parapatry occurred under several conditions, including when both species were identical in dispersal, intrinsic rate of growth, and competition but differed in their spatial performances. Results indicate that overlapping distributions and parapatry are equally expected for close species. Moreover, similar species in parapatry tend to exhibit equivalent range sizes. This model explains how species can coexist regionally while maintaining spatial exclusion. It also describes how a species that is rare in distribution can invade the range of a similar and widespread species. We discuss the limitations of using present species distributions for recognizing modes of speciation, and we suggest studying more extensively the relationship between density-dependent dispersal and interspecific competition. We show that densitydependent dispersal can favor segregation.
\end{abstract}

Key words: coexistence of species; density-dependent dispersal; dispersal vs. competition; invasion; Lotka-Volterra model, spatial variation; modeling parapatry in closely related species; parapatry, spatial model; sharp boundaries; spatial variation; species distributions; species segregation.

\section{INTRODUCTION}

Many species are spatially separated by sharp boundary ranges. The distribution of these species ranges is contiguous and without any physical barriers between them. This spatial segregation, or parapatric distribution, is a widespread phenomenon both taxonomically and geographically (Bull 1991), and is a common form of distribution between closely related species (Anderson and Eversen 1978, King 1993). A comparison of range distributions for 130 pairs of sibling species resulted in $41 \%$ parapatry, $41 \%$ overlap, and $18 \%$ separate ranges (Anderson and Eversen 1978). Parapatry is the geographic pattern that is assumed to result from parapatric speciation, allopatric speciation with secondary contact, or peripheral isolated speciation (White 1973, Bush 1975, Endler 1977, Lynch 1989). Although

Manuscript received 2 June 1998; revised 14 January 1999; accepted 19 January 1999.

${ }^{4}$ Address correspondence to this author.

E-mail: maini@maths.ox.ac.uk

${ }^{5}$ Present address: Instituto de Zoología Tropical, Facultad de Ciencias, Universidad Central de Venezuela, Apartado 47058, Caracas 1041-A, Venezuela. in some cases narrow hybrid zones occur, parapatric boundaries without a hybrid zone have been closely mapped in several studies (e.g., Thomomys talpoides complex [Thaeler 1974], Ranidella frogs [Bull 1991], Proechimys rodents [García 1981], and Sorex shrews [Neet and Hausser 1990]). Spatial segregation occurs also in species of different genera but with similar ecological requirements (e.g., the barnacles Chthamalus and Balanus [Connell 1961], and the ticks Aponomma and Amblyomma [Bull 1991]). Parapatry without hybridization and in less-related species suggests that ecological factors could explain this spatial pattern. In this paper we study an ecological mechanism for predicting parapatry in similar species. Closely related species are nearly alike in morphology and ecological requirements, especially in the case of chromosomal species that are almost identical. Most explanations of parapatric distributions assume that competitive interactions and species similarity can cause interspecific exclusion upon geographic contact (Chappell 1978, Terborgh 1985, Haffer 1989, Neet and Hausser 1990). Environmental gradients appear also to affect the distribution of contacting species, and parapatric bound- 
aries occur following gradients in elevation, climate, soil structure, and vegetation (Mayr 1963, Miller 1967, MacArthur 1972, States 1976, Terborgh 1985, Kohlmann et al. 1988, Bull 1991, Bull and Possingham 1995).

Patterns of distribution for two competitive species have been modeled for continuous and discrete space (McLaughlin and Roughgarden 1993). In addition to competition and environmental gradients, theoretical studies in continuous space have tested several other assumptions on growth rate, spatial quality, and mating systems, and included dispersal. However, they commonly predict complete or partial overlap in the species distribution. Models we examined include (a) dispersal in which individuals tend to move towards favorable areas of the environment and away from each other (Shigesada et al. 1979); (b) self-density diffusion and spatially varying growth rates (Namba 1989); and (c) interspecific mating that reduces the species' reproductive success (Ribeiro and Spielman 1986). These and other studies predict only partial segregation with at least some overlap in species distributions (Gopalsamy 1977, Shigesada et al. 1979, Mimura and Kawasaki 1980, Namba and Mimura 1980, Okubo 1980, Murray 1989, Namba 1989, Bull and Possingham 1995, Shigesada and Kawasaki 1997).

Total segregation can occur if one considers a diffusion mechanism in which each species disperses towards places within a finite habitat where the total population density is at a minimum (Bertsch et al. 1984, Gurtin and Pipkin 1984, Bertsch et al. 1985, Bertsch et al. 1987). In their studies the above authors do not consider any interaction terms, and they deal only with different aspects of the spatial dynamics. For instance, Bertsch et al. (1984), looked for initial conditions for which a sedentary species forms a barrier for a dispersive species. They found that an initially segregated distribution would remain segregated. A general diffusion term dependent on total density that preserves segregation has been studied by Witelsky (1997). Lui and Keller (1987) studied the effect of adding a growth term to the dispersive species while the second species was considered as sedentary. In our study, we are interested in segregated distribution for general situations, where both species disperse and grow, regardless of the size and initial location of the species.

We investigate the spatial patterns of two competing species. We ask the questions: Can two similar species coexist in equilibrium and show spatial segregation? Furthermore, can a rare species expand into the range of a similar species when both are dispersing, resulting in a stationary parapatric boundary? Our model consists of two reaction-diffusion equations for species densities. The diffusion results from a gradient proportional to the sum of the densities of both species. The competitive interactions are of Lotka-Volterra type, and a heterogeneous environment reduces the population growth rate. Our equations differ from pre- viously mentioned work on segregation because they consider dispersal and growth terms for both species as well as a spatially varying environment. Two analyses were conducted: a phase-plane study to investigate the solutions in the absence of dispersal, and numerical simulations to solve the full system. We discuss the ecological implications of our results.

\section{THE ModeL}

The model considers two interacting species on the finite one-dimensional domain scaled to $[0,1]$ with densities $u_{1}(x, t)$ and $u_{2}(x, t)$ at position $x$ and time $t$, with spatiotemporal dynamics described by the following partial differential equations:

$$
\begin{aligned}
\frac{\partial u_{1}}{\partial t}= & D_{1} \frac{\partial}{\partial x}\left[u_{1} \frac{\partial}{\partial x}\left(u_{1}+u_{2}\right)\right] \\
& +r_{1} u_{1}\left[1-f_{1}(x)-\frac{\alpha_{11} u_{1}+\alpha_{12} u_{2}}{k_{1}}\right] \\
\frac{\partial u_{2}}{\partial t}= & D_{2} \frac{\partial}{\partial x}\left[u_{2} \frac{\partial}{\partial x}\left(u_{1}+u_{2}\right)\right] \\
& +r_{2} u_{2}\left[1-f_{2}(x)-\frac{\alpha_{21} u_{1}+\alpha_{22} u_{2}}{k_{2}}\right] .
\end{aligned}
$$

The first term on the right-hand side (RHS) of Eqs. 1 and 2 models dispersal, where each species disperses towards places with lower total population density with a flux proportional to the gradient of the total density, $\partial / \partial x\left(u_{1}+u_{2}\right) . D_{i}$ is the diffusion constant of species $i$. This form of the dispersal term was derived by Gurtin and Pipkin (1984) using the mass conservation law and the assumption that the dispersal velocity is proportional to the gradient of the total population density. The form for the macroscopic population motion term in reaction-diffusion equations is based on the assumptions made concerning the factors influencing an individual's behavior to move. This term can be derived using a continuous approximation of a discrete randomwalk process or by an argument based on fluxes as in our model (see derivations in Okubo [1980], Nisbet and Gurney [1982], Edelstein-Keshet [1988], Murray [1989], and especially Turchin [1998: Appendix A]). The second term on the RHS describes the interaction. It includes (a) competition that is similar to the LotkaVolterra type, where $\alpha_{i j}$ is the competition coefficient that defines the effect of species $j$ on the growth rate of species $i$, and $k_{i}$ (assumed equal to 1 ) is the largest carrying capacity; and (b) relative growth rate, which is spatially dependent, $1-f_{i}(x)$. The space dependence in the interaction terms may describe gradients in soil type, moisture, elevation, or temperature, which could impose a reduction on the growth. The performance of species 1 is considered highest at $x=0$ and declines towards $x=1$, while the performance of species 2 shows the opposite trend. The reduction in the species' performance is described by $f_{1}(x)=a_{1} x^{h}$ and $f_{2}(x)=$ 


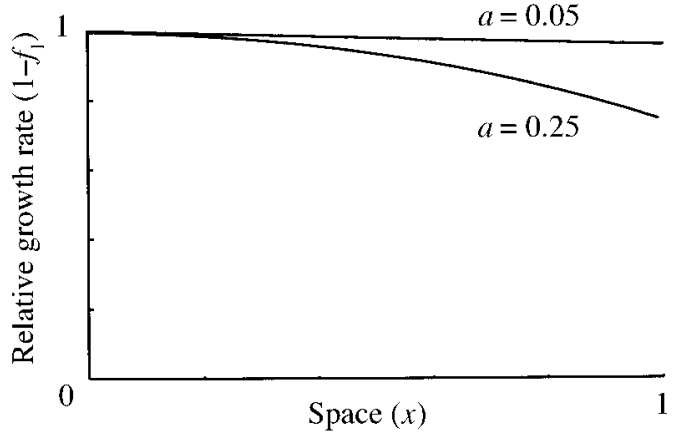

FIG. 1. Effect of two degrees of reduction in performance, $a=0.05$ and 0.25 , on the relative spatial growth rate for species $1,1-f_{1}(x)$.

$a_{2}(1-x)^{h}, h \geq 1$, where $x$ varies within the interval $[0,1]$. The model was analyzed for $h=2$. The extent of growth reduction is measured by $a_{i}$ with values between 0 and 1 . If $a_{i}=0$, then species $i$ is well adjusted everywhere and the spatial domain is homogeneous for it. If $a_{i}>0$, then the performance of species $i$ is space dependent (Fig. 1).

Several studies have documented spatial variation in species performance (Pulliam 1996). Field researchers have shown that in peripheral populations of birds, death rates exceed birth rates (Wiens and Rotenberry 1981). García-Ramos and Kirkpatrick (1997) determined that asymmetrical gene flow along clines may prevent populations from evolving to their local ecological optima. Kirkpatrick and Barton (1997) demonstrated how maladaptations towards peripheral populations may limit the species distribution. We choose a quadratic form for $f_{i}(x)$ because it is consistent with results on spatial decay in fitness (Kirkpatrick and Barton 1997). The intrinsic rate of increase is denoted $r_{i}$. The domain has reflecting boundaries for both species, a condition satisfied when $u_{1} \partial / \partial x\left(u_{1}+u_{2}\right)=0$ and $u_{2} \partial / \partial x\left(u_{1}+u_{2}\right)=0$. The biological interpretation of this condition is that neither species leaves the domain. This model focuses on the situation where the species are similar in many ways, $a_{1}=a_{2}=a, \alpha_{11}=\alpha_{22}=1$, $\alpha_{21}=\alpha$ and $\alpha_{12}=m \alpha$, but they can differ in their response to the environment $f_{i}(x)$, where $m$ describes the similarity between the interspecific competition coefficients, $m \leq 1$. We assume no hybridization between species. These simplifications allow us to focus on the roles of density-dependent diffusion, interspecific competition, and spatially varying performance. All the parameters appearing in Eqs. 1 and 2 are positive constants.

\section{RESULTS}

Spatial dynamics without dispersal: Graphical analysis

The model, Eqs. 1-2, is a fully nonlinear coupled set of partial differential equations. As such, it is not readily amenable to an analytic treatment. Therefore, we first studied the equilibria densities under competition in a heterogeneous environment in the absence of dispersal. This provides valuable insight to the effects of the spatially varying terms in the kinetics. For no dispersal in Eqs. 1 and 2, the isoclines for $u_{1}$ and $u_{2}$, which correspond to those sets of $\left(u_{1}, u_{2}\right)$ satisfying $\partial u_{1} / \partial t=\partial u_{2} / \partial t=0$ are given, respectively, by

$$
\hat{u}_{2}=\frac{1-a x^{2}-\hat{u}_{1}}{m \alpha}
$$

and

$$
\hat{u}_{2}=1-a(1-x)^{2}-\alpha \hat{u}_{1} .
$$

For fixed $x$, the isoclines are linear; $x$ determines where the isoclines intersect the $u_{2}$ axis: as $x$ increases, isocline 1 moves down while isocline 2 moves up (Fig. $2)$. There are three nontrivial (and nonnegative) equilibria, $E$, for each locality $x: E=\left\{\left(\hat{u}_{1}, \hat{u}_{2}\right),\left(\hat{u}_{1}, 0\right),(0\right.$, $\left.\left.\hat{u}_{2}\right)\right\}$. They depend on the values of $a, \alpha, m$, and the initial density distributions (Table 1, Fig. 3).

There are two conditions that lead to parapatry, besides particular initial species densities. The first condition is no local coexistence, which requires that isocline 2 has a lower slope than isocline 1 (Roughgarden 1979). This occurs for $m>1 / \alpha^{2}$, which means that one
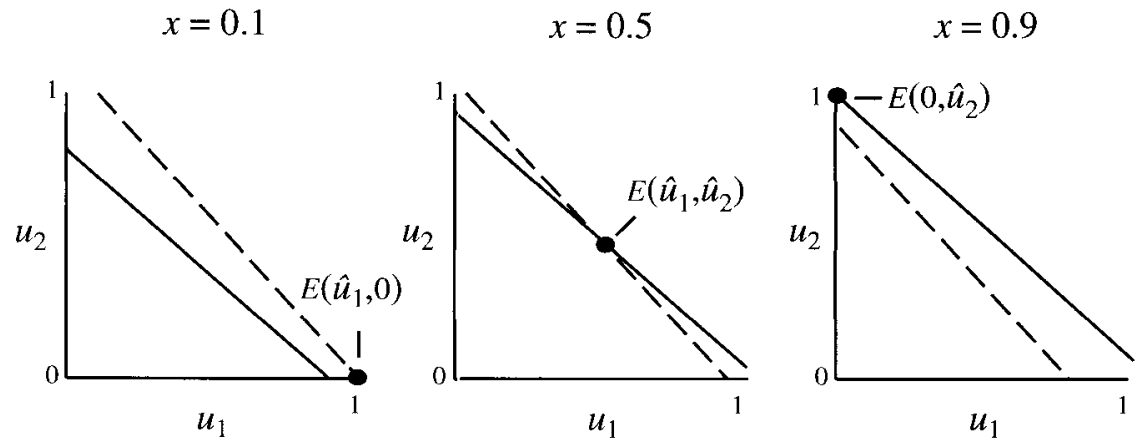

FIG. 2. Spatial effects on the relative positions of the isoclines for two competitive species. Isoclines for $u_{1}$ (dashed line, Eq. 3) and for $u_{2}$ (solid line, Eq. 4) are shown. Localities are $x=0.1,0.5$, and 0.9. Parameters were $a=0.25$ and $\alpha=m \alpha$ $=0.9$. The stable equilibria for the species densities are denoted by $E$. 
TABLE 1. Effect of the reduction in performance $(a)$, and the interspecific competition coefficients $(\alpha$ and $m)$, on spatial equilibrium densities for two species, $\hat{u}_{1}(x)$ and $\hat{u}_{2}(x)$, from Eqs. 1 and 2 for zero dispersal. Results are illustrated in Fig. 3.

\begin{tabular}{lccc}
\hline \hline \multicolumn{1}{c}{ Parameters } & Interval $x$ & $\hat{u}_{1}(x)$ & $\hat{u}_{2}(x)$ \\
\hline$a=0$ & & $(1-m \alpha)$ & $(1-\alpha)$ \\
$\alpha, m \alpha<1$ & {$[0,1]$} & $\frac{\left(1-m \alpha^{2}\right)}{\left(1-m \alpha^{2}\right)}$ & 0 \\
$\alpha \geq 1, m \alpha<1$ & {$[0,1]$} & 1 & $1-\hat{u}_{1}(x)$ \\
$\alpha=m \alpha=1$ & {$[0,1]$} & {$[0,1] \dagger$} & $1-\hat{u}_{1}(x)$ \\
$\alpha, m \alpha>1$ & {$[0,1]$} & 1 or $0 \dagger$ & 0 \\
$a>0$ & {$\left[0, x_{a}\right]$} & $1-a x^{2}$ & $1-a(1-x)^{2}-\alpha+a \alpha x^{2}$ \\
$m \alpha^{2}<1$ & $\left(x_{a}, x_{b}\right)$ & $\left(1-m \alpha^{2}\right)$ \\
& {$\left[x_{b}, 1\right]$} & 0 & $1-a(1-x)^{2}$ \\
& {$\left[0, x_{b}\right)$} & $1-a x^{2}$ & 0 \\
$m \alpha^{2} \geq 1$ & {$\left[x_{b}, x_{a}\right] \dagger$} & $1-a x^{2}$ & 0 \\
& $\left(x_{a}, 1\right]$ & or $0 \dagger$ & $1-a(1-x)^{2}$ \\
& & 0 & $1-a(1-x)^{2}$ \\
\hline
\end{tabular}

Notes: In the "Interval" column, $x_{a}$ is the spatial location where isoclines intercept at coordinates $\left(u_{1}, 0\right)$, and $x_{b}$ is the location at which the interception occurs at coordinates $\left(0, u_{2}\right)$. Definitions are:

$$
x_{a}=\frac{1-\sqrt{\frac{(\alpha-1)^{2}}{a}+\alpha}}{1-\alpha} \quad x_{b}=\frac{-m \alpha+\sqrt{\frac{(m \alpha-1)^{2}}{a}+m \alpha}}{1-m \alpha} .
$$

For $x_{i}<0$ or $x_{i}>1, x_{i}$ is set to be 0 or 1 respectively.

+ Densities at each $x$ depend on the initial conditions.

\$Densities do not apply for $m \alpha^{2}=1$; for these values the isoclines are coincident at $x_{a}=x_{b}$, and for $\alpha=m \alpha=1$ the isoclines are coincident at $x=1 / 2$. Spatial patterns are shown in Fig. 3.

interspecific competition coefficient should be higher than $1(\alpha>1)$ to have $m \leq 1$. The second condition is regional coexistence, which, considering the symmetry in our model, is given if at the best locality for species 2 , isocline 2 is above isocline 1 . That is, if at $x=1$ the intercept on the ordinate $u_{2}$ is higher for isocline 2. This occurs for $m \alpha>1-a$, and indicates that the second interspecific coefficient can be lower than 1. It shows, in addition, how the spatial variation favors global coexistence and parapatry for unequal interspecific competition. We next discuss the spatial patterns in detail.

In the simple case, when there is spatial homogeneity in performance (i.e., when $a=0$ ) all the localities behave identically, and coexistence or extinction can occur depending on the strength of the interspecific competition coefficients $\alpha$ and $m \alpha$. This situation follows the typical Lotka-Volterra result but is extended to many localities (Roughgarden 1979). Therefore, depending on the values of the parameters $m$ and $\alpha$, there are four results: (a) for $\alpha$ and $m \alpha<1$, there is local stable coexistence, resulting in overlapped species distributions; (b) for $\alpha=m \alpha=1$, there are neutral equilibria with local species coexistence depending upon initial densities; (c) for $\alpha$ and $m \alpha>1$, coexistence no longer occurs and the species dominant in each locality also depends upon initial densities; (d) for $\alpha>1$ and $m \alpha<1$ the stronger competitor excludes the weaker species. In addition to local noncoexistence, parapatry requires for the case without dispersal that initial distributions favor each species within a continuous region. For example, segregated initial conditions result in parapatry because each species exhibits a continuous and separate range.

When there is spatial heterogeneity in performance, that is $a>0$, the spatial locality drives the relative position of the isoclines, and three regions that are qualitatively different may appear. In the extreme region that includes $x=0$, isocline 1 is generally above isocline 2, so species 1 excludes species 2 (Fig. 2). At the other extreme, with $x=1$, isocline 2 tends to be above isocline 1, and species 1 is excluded. At the center the isoclines can cross and the outcome here depends again upon the intensity of interspecific competition. The center is situated between localities $x_{a}$ and $x_{b}$ (Table 1). This center region shares the same results with the case without spatial variation for both interspecific competition coefficients lower or higher than 1. This region, however, differs for $\alpha>1$ and $m \alpha<$ 1 where the region can maintain both species depending on the extent of the spatial variation: (a) for $m \alpha^{2}<1$ the species may coexist in each locality within the re- 
$\alpha=m \alpha=0.9$
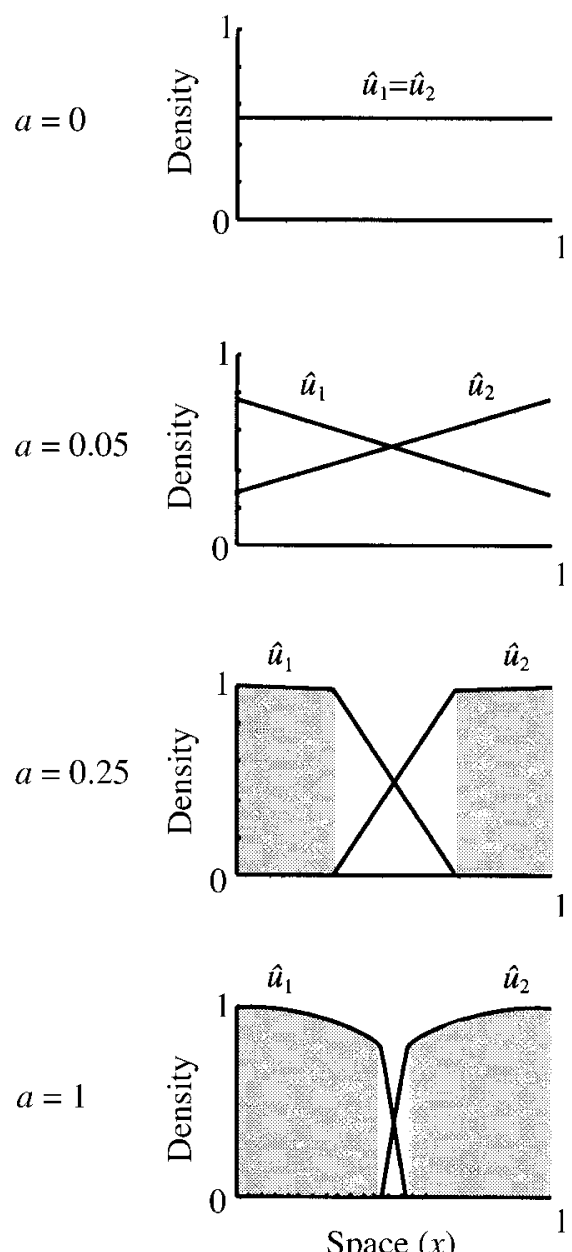

$\alpha=m \alpha=1.0$
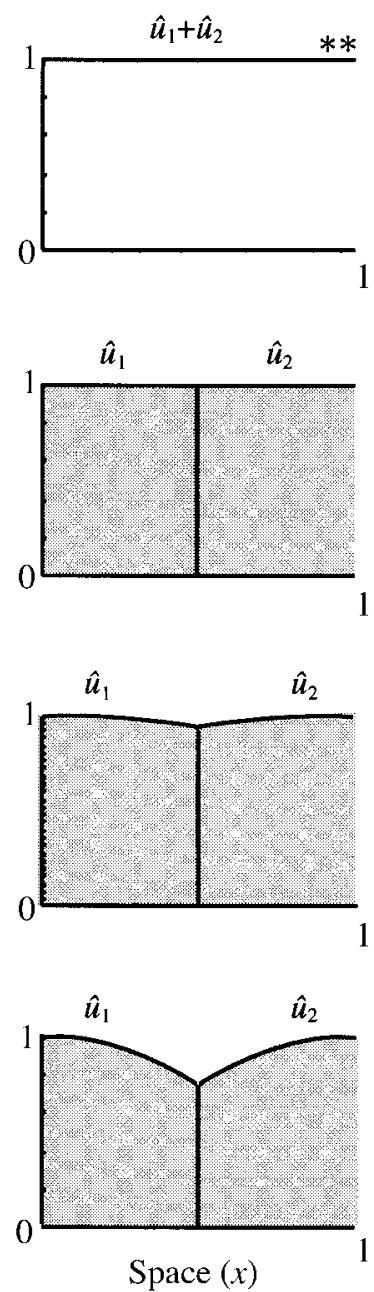

$\alpha=m \alpha=1.1$
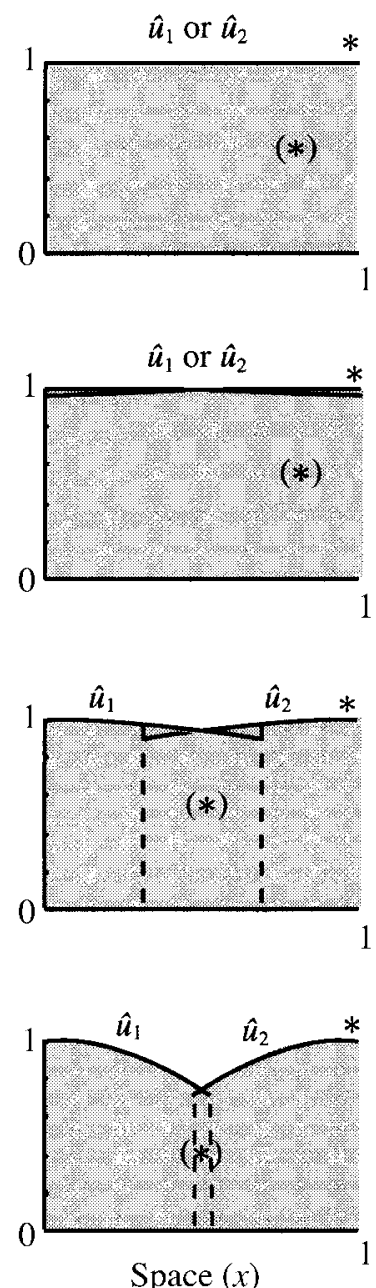

FIG. 3. Spatial patterns for species densities from Eqs. 1 and 2 with zero diffusion. These analytical results (see Table 1) illustrate the effect of the interspecific competition coefficients $\alpha$ and $m \alpha$ (increasing from left to right) and the reduction in performance $a$ (decreasing from bottom to top) on equilibrium densities of two species competing in isolated localities. Shaded areas indicate nonoverlapping distributions. Parapatry requires nonoverlap and continuous species distribution throughout space. Asterisks refer to initial conditions: $* *=$ results only for segregated initial conditions; $*=$ results also for some overlapped initial densities in the domain. For example, equal initial densities show parapatry with the boundary at the center of the domain. The initial densities that let a species dominate the locality $x$ within the region $(*)$ are given by the particular phase plane for $x$.

gion if $\alpha<1 /(1-a)$. This condition results from considering that at the best locality for species $2(x=$ 1 ), the intercept on the abscissa $u_{1}$ is higher for isocline 2 ; (b) For $m \alpha^{2}>1$ and $m \alpha>1-a$ there is regional but no local coexistence, with results depending on initial densities. This case was mentioned above in requirements for parapatry (see regions in Fig. 3, columns 1 and 3, rows 3-4). When $m \alpha^{2}=1$ the isoclines run parallel for each $x$, and are coincident for $x_{a}=x_{b}$. At each side of that point, a different species dominates the spatial domain. This is considered to be a parapatric distribution. Global coexistence for this last case requires that isocline 2 is above isocline 1 , at least at the best locality for species 2 . That is to say, the intercepts on the abscissa and the ordinate should be higher for isocline 2 at $x=1$. This is satisfied for $1 / \alpha=m \alpha>$ $1-a$.

This analysis, in the absence of dispersal, shows that parapatry is favored under several conditions. It results in a stable solution when $a>0, m \alpha^{2}=1$ and $m \alpha>$ $1-a$, if both species exist initially everywhere at any density (Fig. 3, column 2, rows 2-4). Parapatry could appear for other conditions, $m \alpha^{2}>1$ and $m \alpha>1-$ $a$, depending upon the particular initial distributions. The existence of an abrupt zonation has been described in studies on competition along environmental gradients. These models are also dispersal-free and considered the competition coefficients equal to 1 . Pielou 


$$
\alpha=m \alpha=0.9
$$
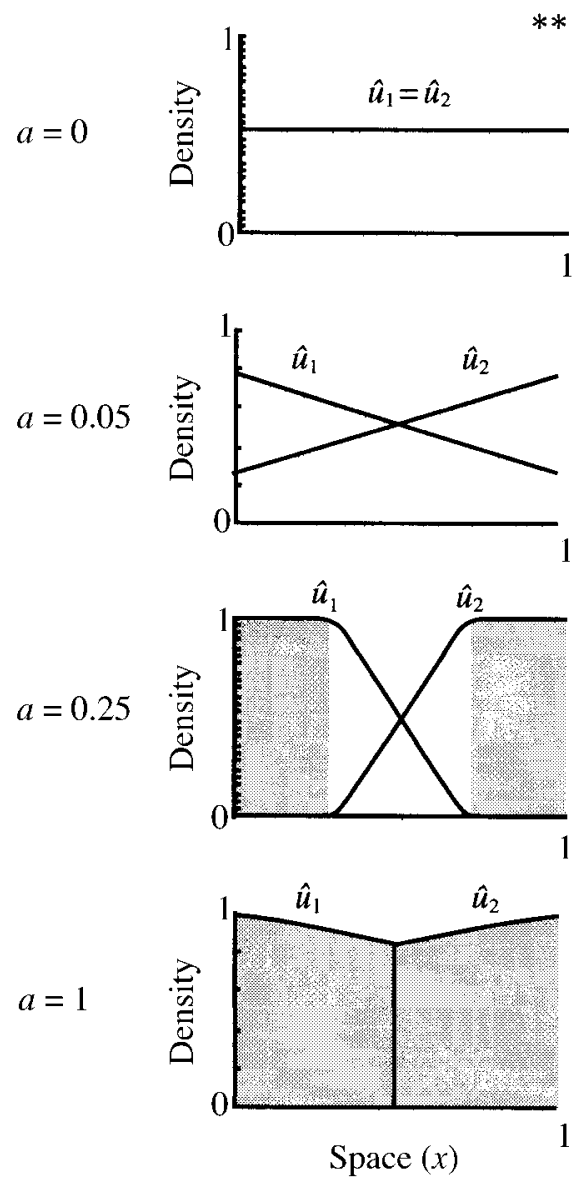

$\alpha=m \alpha=1.0$
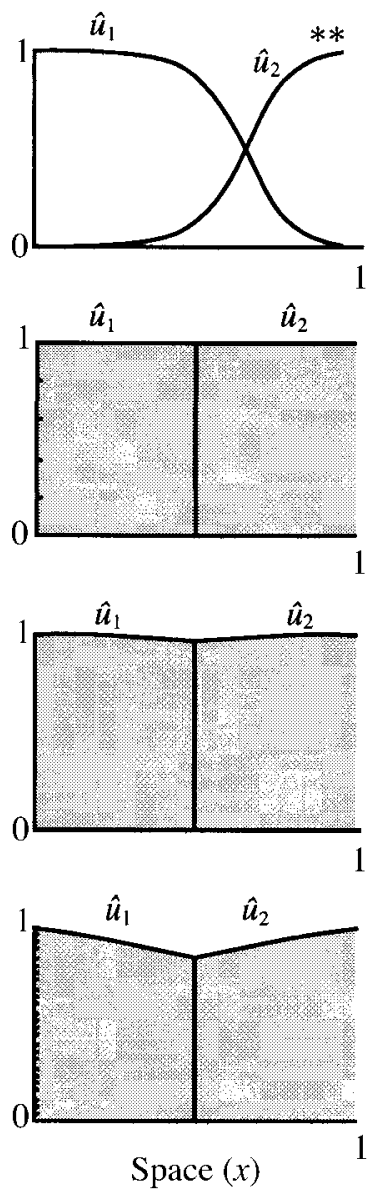

$\alpha=m \alpha=1.1$
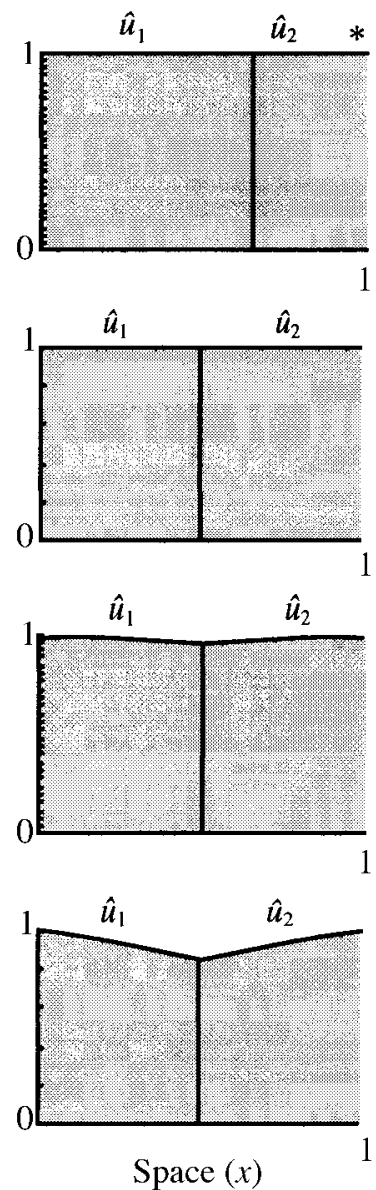

FIG. 4. Results of numerical simulations for species distribution for the full model. Effect of the performance reduction, $a$, and the competition coefficients, $\alpha$ and $m \alpha$, on equilibria densities for two competing species. Parameters and initial distributions were $r_{1}=r_{2}=0.3, D_{1}=D_{2}=0.005, u_{1}(x, 0)=0.3 \exp \left[-(x-0.5)^{2} / 0.04\right]$ and $u_{2}(x, 0)=0.3 \exp \left[-(x-0.8)^{2 /}\right.$ 0.04]. Initial conditions that may result in parapatry for $a=0$ are denoted by $*$ and $* *$ (see Fig. 3 for details). The appearance of sharp solutions for Eqs. 1 and 2 produces oscillations in the numerical solution around the point at which the derivative is discontinuous (Finlayson 1992). Oscillations do not appear to affect our results, and they were averaged using a standard method. More sophisticated numerical procedures would allow more efficient simulation of these types of solutions.

(1974), and MacLean and Holt (1979), described parapatry when the relative carrying capacity of the species varies along a gradient. Similarly Endler (1977: 78) described a cline of width zero for two morphs of a species when selection depends on the spatial position. Our dispersal-free case differs because the growth rate is the parameter that depends on space.

\section{Simulations with dispersal}

When dispersal is considered and the performance of the two species is allowed to respond to the spatial gradient $(a>0)$, segregation and range overlap may result depending on the strength of the interspecific competition coefficients and the degree of the spatial variation (Fig. 4). Simulations displayed two main results: (a) for both coefficients $\geq 1$ there is spatial seg- regation ( $\alpha, m \alpha \geq 1$, Fig. 4, column 2-3, rows 2-4); (b) for only one coefficient, or both coefficients $<1$, the outcomes depend on $a$. In both cases species exhibit overlapping ranges that decrease towards spatial segregation as $a$ goes to 1 (Fig. 4, column 1, row 4; Fig. 7).

The model was solved numerically using the D03PPF routine of the NAG (Numerical Algorithms Group) Fortran Library (NAG 1999). The spatial discretization used finite differences, and the method of lines was employed to reduce the partial differential equations to a system of ordinary differential equations (Ames 1992). Automatic remeshing for 1500 spatial points was considered. Simulations for several overlapping and nonoverlapping initial distributions converged to the same stationary species distributions. 


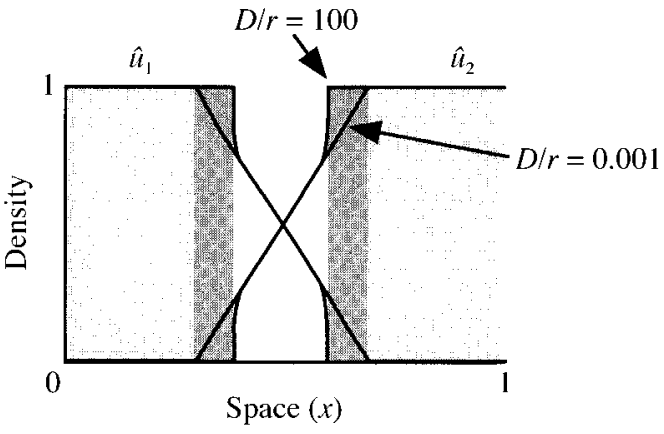

FIG. 5. Effect of dispersal coefficient relative to growth rate on the extent of species overlap. Parameter values were identical for $D$ and $r$, so $D_{1} / r_{1}=D_{2} / r_{2}=D / r$. The arrows indicate species distribution for two values of $D / r$ : low ratio $(D / r=0.001)$, and large ratio $(D / r=100)$. Darker-shaded areas show the additional nonoverlapped localities for the large ratio. Parameters were $a=0.25$ and $\alpha=m \alpha=0.9$.

These numerical simulations suggest that the distributions for $a>0$ could be globally stable. These initial conditions included situations that may resemble a species invasion. This occurred when the resident species was close to its carrying capacity for most of the spatial domain, and the invading species was rare in abundance and/or distribution. For example, for an invading species that is initially segregated from the resident species, the initial densities were $u_{1}(x, 0)=\exp \left[-x^{2} / 4 \times\right.$ $\left.10^{-5}\right]$ and $u_{2}(x, 0)=0$ for the interval $x \in[0,0.003]$; and $u_{1}(x, 0)=0$ and $u_{2}(x, 0)=\exp \left[-(x-0.5)^{8 / 0.04}\right]$; for the rest of the domain $(0.003,1]$. For an invading species initially overlapping with the resident, for $x \in$ $[0,0.003]$ population densities were $u_{1}(x, 0)=0.4$ $\exp \left[-x^{2} / 4 \times 10^{-5}\right]$ and $u_{2}(x, 0)=0.6 \exp \left[-(x-0.5)^{8 /}\right.$ $0.04]$; and $u_{1}(x, 0)=0$ and $u_{2}(x, 0)=\exp \left[-(x-0.5)^{8 /}\right.$ $0.04]$ for the rest of the domain. Parameters studied include the case when species are identical in many aspects, $\alpha=m \alpha, D_{1}=D_{2}$ (i.e., the diffusion coefficients are equal), and $r_{1}=r_{2}$ (i.e., the intrinsic growth rates are equal). The parameter values were chosen by considering their biological relevance. Some mobile species disperse distances equivalent to several percentages of their range (García-Ramos and Kirkpatrick 1997). For a random dispersal, $\sigma^{2}$ is the dispersal variance, and the mean distance between the place of birth of an individual and its mother is $\sim 0.8 \sigma$. Values of $\sigma^{2}$ $=5 \times 10^{-5}$ and $\sigma^{2}=0.005$, standardized by the species range size, correspond to individual dispersal distances of $0.6 \%$ and $5.7 \%$ of its range, respectively. For a total density-dependent dispersal, we do not have estimates of $D_{i}$, so we considered $\sigma^{2}$ as an approximation of its magnitude. Data on maximal rates of increase range from 0.1 to 8 (Pianka 1988); we selected the common values of $r_{i}=0.3$ and $r_{i}=0.9$.

Results indicated that parameter values may modify the spatial pattern. Large diffusion coefficients or small growth rates reduce species overlap. Their joint effects are described by a large ratio between dispersal coef- ficients and growth rates. This case, for species identical in these parameters, showed a moderate reduction in overlap $\left(D_{1} / r_{1}=D_{2} / r_{2} \geq 1\right.$, Fig. 5). When the ratio is low $\left(D_{i} / r_{i} \ll 1\right)$, the results resemble the case without dispersal and the species evolve to spatial distributions with a high degree of overlap. Furthermore, a differentiation between species for the values of the dispersal coefficients or growth rates will affect the symmetry in the species distributions. The species with a larger dispersal homogenizes its population densities to a greater degree. The species with a larger growth rate increases its population densities. Both modify the density landscape and move the minimum for total density far from the central range. With parapatry, the boundary follows the new minimum location, and the faster disperser or riser will enlarge its range. Moderate change in range sizes was observed when parameters differed by a factor of 10 (Fig. 6). For the cases with identical dispersal and growth rates the parapatric boundary is located at the center of the spatial domain even for asymmetry in the interspecific competition (Fig. 7). Other elements in the model can also differ between species, such as $a_{i}$, or symmetry in $f_{i}(x)$, which could also modify the symmetry in the results. We do not consider all species differences or their combinations; rather, we focus on cases where species show similitude.

When there is spatial homogeneity in performance $(a=0)$, segregation could result depending upon interspecific coefficients and the initial distributions.
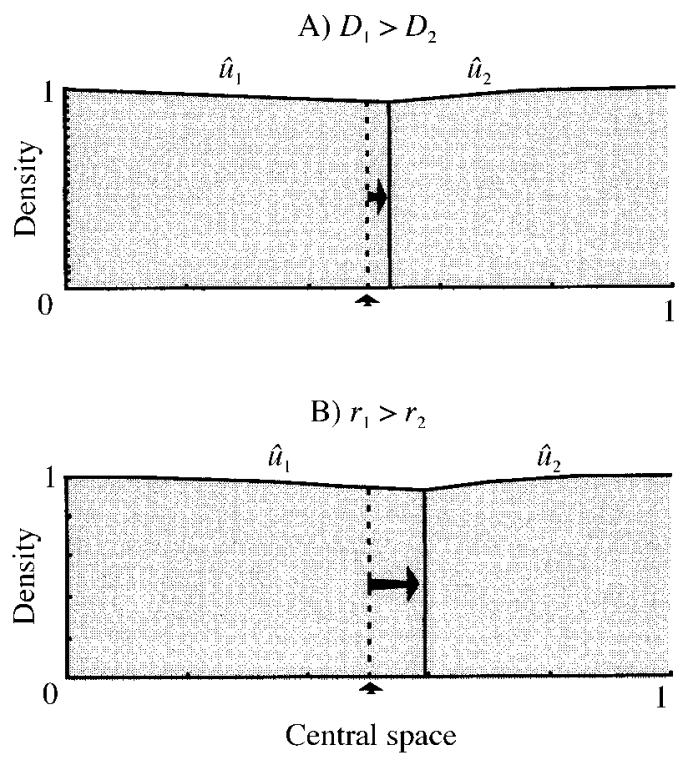

FIG. 6. Effect of differences between species in parameter values on location of parapatric boundary. (A) Unequal dispersal coefficients: $D_{1}=0.005, D_{2}=0.0005$ with $r_{1}=r_{2}=$ 0.3 . (B) Unequal intrinsic growth of increase: $r_{1}=0.9, r_{2}=$ 0.3 with $D_{1}=D_{2}=0.005$. Other parameters were $\alpha=m \alpha$ $=1$ and $a=0.1$. Initial conditions were as in Fig. 4. The vertical dotted line indicates central range and boundary location for identical dispersal and growth rates. 


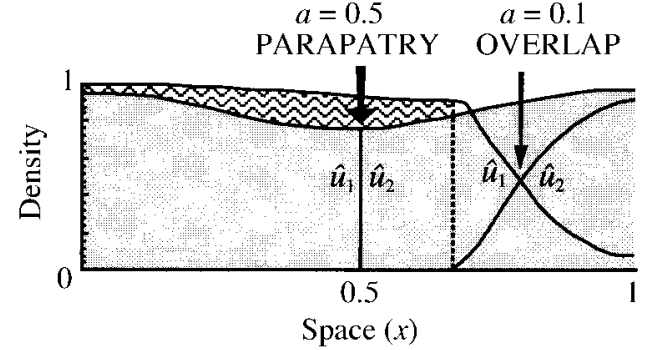

FIG. 7. Effect of the spatial performance and unequal interspecific competitions on spatial patterns. Asymmetrical overlap for $\alpha=1.25, m \alpha=0.65$, and $a=0.1$ is shown on the right; symmetrical parapatry for $\alpha=1.5, m \alpha=0.2$, and $a=0.5$ is shown on the left. Other parameters were: diffusion $D_{1}=D_{2}=0.005$, and intrinsic growth rates $r_{1}=r_{2}=0.3$.

These particular cases are of limited interest because they do not provide an explanation for how a species advances into occupied areas.

The isocline and full system analyses show similar patterns when there is variation in performance (Figs. 3 and 4). There are two notable differences between the analyses. The full equations exhibited stable segregated distributions for the following cases: (a) for $m \alpha^{2}>1$ and $m \alpha>1-a$, where dispersal on a heterogeneous space allows one species to successfully invade localities occupied by the other species. This situation provides stability because it overcomes the effect of the initial conditions (Fig. 4, column 3, rows 2-4); (b) for $m \alpha^{2}<1$, where dispersal reduces species overlap and may result in parapatry for large $a$ (Fig. 4, row 3, column 1; Fig. 7). These results suggest that the effect of adding density-dependent dispersal is to sharpen parapatry in situations where one expects either coexistence or priority effects to determine the dominant species for local competitive interactions in the limit of low dispersal rates.

\section{Simulations for alternative dispersal modes}

For an illustration of the effect of the type of dispersal mode on the species distributions, we compared our model with: (a) random dispersal, and (b) the case where dispersal of a species is dependent only on its own density, for particular conditions. For the first mode, the dispersal is defined by $D_{i} \partial^{2} u_{i} / \partial x^{2}$, which replaces the diffusion term of Eqs. 1 and 2. For the second mode, dispersal is $D_{i} \partial / \partial x\left(u_{i} \partial u_{i} / \partial x\right)$. For the purpose of comparison, $D_{i}$ is assumed identical for the three situations. The simulations show substantial overlap in the distributions for both dispersal modes (Figs. 8A, B), unlike the case with total density-dependent dispersal, which leads to entire segregation (Fig. 8C).

\section{DISCUSSION}

Parapatry describes a geographic pattern in which the ranges of two species have separate but contiguous distributions. This study focused on an ecological mechanism to predict parapatry in similar species that involve total density-dependent dispersal, competition, and spatial quality. Parapatric distributions are predicted in our full model for several situations (Table 2): (a) in the simplest case where both species are identical in dispersal, growth rate, and competition parameters, but there is at least a minute variation in their spatial performances. Stable parapatry also occurred when species differ in competition for either (b) interspecific competition higher than intraspecific, or (c) interspecific competition lower than intraspecific but with high spatial variation in performance. This model explains how similar species can share the space while maintaining spatial segregation, but, crucially, it differs from previous work because it predicts a complete spatial segregation. These results are independent of initial conditions, so regardless of whether the species are overlapping or segregated initially, they will segregate at equilibrium.

Stable overlapping species ranges are also predicted. They occur for the case when one or both interspecific competition coefficients are lower than the intraspecific

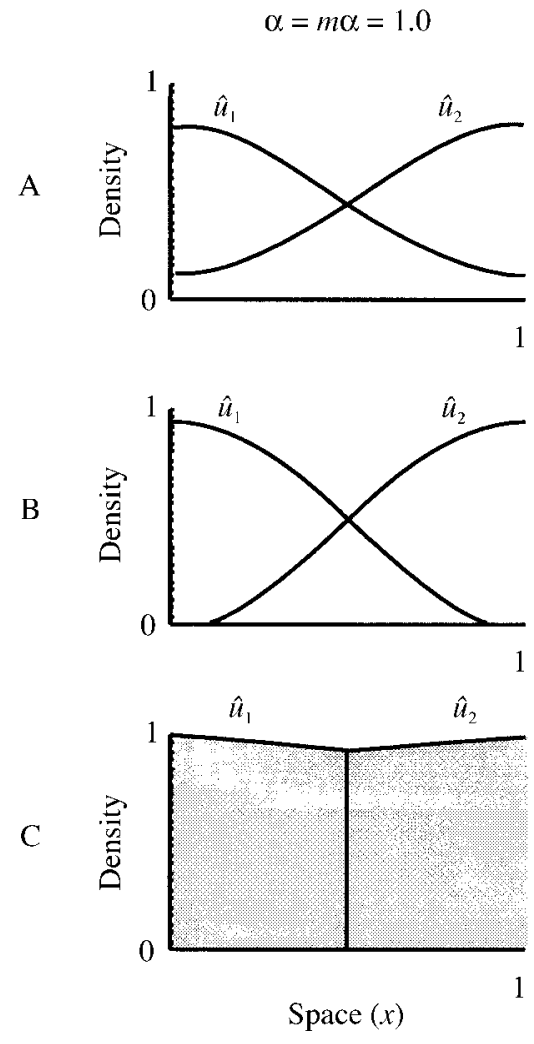

FIG. 8. Effect of the type of dispersal on the pattern of distribution of two species. (A) Each species disperses randomly. (B) Each species disperses depending only on its own density. (C) Each species disperses depending on total densities of both species. Diffusion-term definitions for (A) and (B) are indicated in the text (see Results: Simulations for alternative dispersal modes), and reaction terms are as in Eqs. 1 and $2 ; a=0.5$, and other parameters, initial distributions, and symbols are as in Fig. 4. 
TABLE 2. Summary of the effect of spatial performances and interspecific competition on species distribution for the full model (Eqs. 1 and 2).

\begin{tabular}{lll}
\hline \hline \multicolumn{1}{c}{ Parameters } & Patterns of distributions & Size ranges \\
\hline$a>0$ & & \\
$m \alpha^{2} \geq 1, m \alpha>1-a$ & segregated & approximate $\dagger$ \\
$m \alpha^{2}>1, m \alpha \leq 1-a$; or $m \alpha^{2}<1$ & segregated ( $a$ large $)$ & $\begin{array}{l}\text { approximate } \dagger \\
\text { various }\end{array}$ \\
$a=0$ & & $\ddagger$
\end{tabular}

$\dagger$ Depending on species similarity in growth and dispersal rates. Identical for $D_{1}=D_{2}$ (diffusion coefficients) and $r_{1}=r_{2}$ (intrinsic growth rates). For $m \neq 1, D_{1}=D_{2}$ and $r_{1}=r_{2}$. \$ Segregation or overlap and sizes depend on initial distributions.

ones for varying spatial performance. The amount of overlap decreases incrementally with the reduction in performance, and for a large dispersal coefficient relative to growth rate. Previous models have considered nonlinear diffusion terms or space-dependent interaction. The model presented here combines density-dependent diffusion and space-dependent growth terms. The nonlinearity in the diffusion term of our model makes a general analytical study intractable. Therefore we investigated the system extensively using numerical simulations. From this, we conjecture global stability in certain cases. A future aim is to identify the parameter regime and initial distributions for which a more rigorous analytic study can be performed.

Global stability for these results can explain how a species rare in abundance and distribution can invade the range of a similar and widespread species. The invader can displace, overlap, or partially exclude the established species. For equal dispersal and growth rates, range sizes are identical. For the case of partial exclusion, the parapatric boundary occurs at the middle of the geographic space where the species in expansion is no longer the better competitor and invasion is stopped. When species differ in growth or dispersal rates, the parapatric boundary moves away from the center and the range sizes are unequal. These scenarios could resemble the range establishment for a new species. Invasion of competing species has been studied by several authors (Shigesada 1984, Okubo et al. 1989, Cantrell et al. 1995, Shigesada and Kawasaki 1997: chapter 6). These studies described three outcomes when an invading species spreads into an area occupied by a competitor. The invader completely displaces the resident, they coexist with overlapping, or the invader survives, moving to open spaces that arise eventually. Our model shows a fourth outcome, where the invader partially excludes the resident, resulting in coexistence without overlap.

Connell (1983) compared the relative strengths of intraspecific and interspecific competition. In a quarter of the cases interspecific was stronger than intraspecific competition and in another quarter competitions were identical. He also found that in half of the cases, interspecific was weaker than intraspecific competition.
Using these general statistics, parapatry and overlapped ranges are evenly expected from our analysis. These proportions follow the mentioned observations of Anderson and Eversen (1978).

Alternatively, if the domain is homogeneous concerning species performance, parapatry results depending upon the initial conditions. In this case, the population range also depends on the initial conditions, which means that a small initial range will result in a small stationary range, and a similar species cannot colonize occupied areas. Growth rate and dispersal coefficient values can slightly modify the results. The heterogeneous environment provides stability to the parapatric range. Without it, the parapatric boundary depends on the initial conditions. Furthermore, convergence to equilibria is faster when environmental variation increases. A similar stabilizing effect of a heterogeneous environment has been described for species interactions (Comins and Blatt 1974, Shigesada 1984).

From field research, Haffer (1986) suggested that competition should maintain parapatry even in a region of uniform habitat. Terborgh (1985) and others also pointed out that the distribution of species on environmental gradients will take on a spatially truncated form if the species exhibit competitive exclusion. Our model indicates that competition without or with spatial heterogeneity is quite limited in its ability to explain parapatry. Dispersal is essential to account for this spatial phenomenon, and our results suggest that it must be total density-dependent dispersal. For example, random dispersal in a model with a heterogeneous environment and competition does not predict parapatry, rather it exhibits substantially overlapped distributions.

The effect of population density on dispersal has been well documented for a single species (Denno and Peterson 1995), however, few studies have addressed the effect of interspecific density and the issue of total density-dependent dispersal. Dispersal that depends on total species density has been described for competitive interactions (Lamb and MacKay 1987, Denno and Roderick 1992). In planthoppers, interspecific crowding was found to be as strong a stimulus for the production of migratory forms as intraspecific crowding (Denno 
and Roderick 1992). The effects were reciprocal, with no significative differences. Interspecific effects on alate production are known to occur in aphids as well (Johnson 1965, Lamb and MacKay 1987). If interspecific competition frequently drives dispersal towards places with lower total population density, our model may turn out to be very general.

Our model predicts that the parapatric boundary must occur in the locality of lowest total species density. This prediction matches studies on parapatry with hybrid zones, where hybrid zones that separate species tend to rest in density troughs (Hewitt 1989). Some predictions in our model could be examined using current data: Does parapatry occur even though interspecific competition is lower than intraspecific competition? Are the sizes of parapatric ranges independent of the degree of symmetry in the interspecific competition? To address other predictions empirically, we need to procure two closely related species with the following characteristics: (a) dispersal depending on total density, (b) interspecific competition higher than intraspecific, and (c) different optima along an environmental gradient. We could manipulate an artificial gradient and make it steeper or eliminate it. We could introduce a species and observe whether or not it advances into an already occupied area. We could look at its long-term spatial distribution. However, a qualitative feature of the model should first be addressed if species in parapatry exhibit a dispersal that depends on the density of both species. This can be estimated by examining whether the fraction of disperser increases with the increment of density of both species. Spatial theory may present additional difficulties to be demonstrated empirically. Steinberg and Kareiva (1997) discussed the difficulty in devising clean manipulative experiments to detect the effects of dispersal, as well as in choosing the size of the spatial region for the development of patterning, and to replicate large spatially distributed systems. They suggested evaluating the spatial theory for coexistence and diversity using manipulative experiments, while for spatial phenomena such as stability, invasions, and pattern formation it may be best to combine specific models with largescale or long-term data sets. Furthermore, our model could fit better with those organisms that show densitydependent population regulation produced by space competition or social factors. Sinclair (1989) compared the causes of the density-dependent regulation in several groups of animals. In his review, $8 \%$ of the regulation in insects is caused by emigration of adults due to crowding, in birds $47 \%$ of the regulation is due to social causes, and in small mammals $67 \%$ of the regulation is caused by the exclusion of juveniles from breeding colonies. Species with this type of densitydependent population regulation could also be sensitive to dispersal depending on the density of closely related species.

Some assumptions of our model could be relaxed in further studies. In the model the single factor that determines an individual's movement is the population density. However, in a heterogeneous habitat diverse elements may influence movement. For example, the presence of gradients in fitness may cause a dispersal behavior that moves individuals up in the density gradient. This behavior may favor species displacement towards its higher performance place and could reinforce the spatial segregation. Further analysis must investigate the effect of habitat-choice dispersal. In our model the form for the spatial decay in fitness, $f_{i}(x)$, is well supported. The reduction in fitness, however, may not be continuous and could follow step forms. Also, the shape of $f_{i}(x)$ could affect the speed of the decay of the population density and therefore the impact of the diffusion term on the spatial patterns. Other cases for $f_{i}(x)$ should be explored.

Actual distribution of species has been associated with the geographic speciation modes, neglecting any post-speciational dispersal (Mayr 1963, Lynch 1989). According to Lynch (1989) when close species have large ranges there has been vicariance, and when one species has a smaller range than the other there has been peripheral isolated speciation. He assumed no dispersal and used actual segregated distribution data for close species. Our results show that large segregated ranges may result from diverse initial distributions between similar species. This suggests that species originating through different modes may display the same present distribution. This study supports the arguments that dispersal obscures the geographic pattern of speciation, and present distributions are not a good criterion for recognizing speciation mode (Chesser and Zink 1994).

\section{ACKNOWLEDGMENTS}

We thank Mark Kirkpatrick, Naomi Capuccino, Craig Pease, Art Souther, Jim Bull, and Diego Rodríguez for helpful suggestions. This research (G. García-Ramos) was supported by NSF grant DEB-9407969 to Mark Kirkpatrick. Part of this work (P. K. Maini) was carried out under the Exchange Scheme involving the Academia Mexicana de Ciencias and the Royal Society London.

\section{LiterATURe Cited}

Ames, W. F. 1992. Numerical methods for partial differential equations. Third edition. Academic Press, New York, New York, USA.

Anderson, S., and M. K. Eversen. 1978. Randomness in allopatric speciation. Systematic Zoologist 27:421-430.

Bertsch, M., M. E. Gurtin, and D. Hilhorst. 1987. On interacting populations that disperse to avoid crowding: the case of equal dispersal velocities. Nonlinear Analysis, Theory, Methods and Applications 11:493-499.

Bertsch, M., M. E. Gurtin, D. Hilhorst, and L. A. Peletier. 1984. On interacting populations that disperse to avoid crowding: the effect of a sedentary colony. Journal of Mathematical Biology 19:1-12.

Bertsch, M., M. E. Gurtin, D. Hilhorst, and L. A. Peletier. 1985. On interacting populations that disperse to avoid crowding: preservation of segregation. Journal of Mathematical Biology 23:1-13. 
Bull, C. M. 1991. Ecology of parapatric distributions. Annual Review of Ecology and Systematics 22:19-36.

Bull, C. M., and H. Possingham. 1995. A model to explain ecological parapatry. American Naturalist 145:935-947.

Bush, G. L. 1975. Modes of animal speciation. Annual Review of Ecology and Systematics 6:339-364.

Cantrell, R. S., C. Cosner, and V. Hutson. 1995. Spatially explicit models for the population dynamics of a species colonizing an island. Mathematical Biosciences 136:65107.

Chappell, M. A. 1978. Behavioral factors in the altitudinal zonation of chipmunks (Eutamias). Ecology 59:565-579.

Chesser, R. T., and R. M. Zink. 1994. Modes of speciation in birds: a test of Lynch's method. Evolution 48:490-497.

Comins, H. N., and D. W. E. Blatt. 1974. Prey-predator models in spatially heterogeneous environments. Journal of Theoretical Biology 48:75-83.

Connell, J. H. 1961. The influence of interspecific competition and other factors on the distribution of the barnacles Chthamalus stellatus. Ecology 42:710-723.

Connell, J. H. 1983. On the prevalence and relative importance of interspecific competition: evidence from field experiments. American Naturalist 122:661-696.

Denno, R. F., and M. A. Peterson. 1995. Density-dependent dispersal and its consequences for population dynamics. Pages 113-130 in N. Cappucino and P. Price, editors. Population dynamics. New approaches and synthesis. Academic Press, New York, New York, USA.

Denno, R. F., and G. K. Roderick. 1992. Density-related dispersal in planthoppers: effects of interspecific crowding. Ecology 73:1323-1334.

Edelstein-Keshet, L. 1988. Mathematical models in biology. Random House, New York, New York, USA.

Endler, J. A. 1977. Geographic variation, speciation, and clines. Monographs in Population Biology 10. Princeton University Press, Princeton, New Jersey, USA.

Finlayson, B. A. 1992. Numerical methods for problems with moving fronts. Ravenna Park Publishing, Seattle, Washington, USA.

García, G. 1981. Estudio de una zona de contacto entre los cariomorfos $2 \mathrm{~N}=50$ y $2 \mathrm{~N}=62$ de la superespecie Proechimys guairae (Rodentia: Echimyidae). Dissertation. Universidad Simón Bolívar, Caracas, Venezuela.

García-Ramos, G., and M. Kirkpatrick. 1997. Genetic models of adaptation and gene flow in peripheral populations. Evolution 51:21-28.

Gopalsamy, K. 1977. Competition, dispersal and coexistence. Mathematical Biosciences 33:25-33.

Gurtin, M. E., and A. C. Pipkin. 1984. A note on interacting populations that disperse to avoid crowding. Quarterly of Applied Mathematics 42:87-94.

Haffer, J. 1986. Superspecies and species limits in vertebrates. Zeitschrift für Zoologische Systematik und Evolutionsforschung 24:169-190.

Haffer, J. 1989. Parapatric bird species of the paleartic region. Journal of Ornithology 130:475-512.

Hewitt, G. M. 1989. The subdivision of species by hybrid zones. Pages 85-110 in D. Otte and J. A. Endler, editors. Speciation and its consequences. Sinauer, Sunderland, Massachusetts, USA.

Johnson, B. 1965. Wing polymorphism in aphids. II. Interaction between aphids. Entomologia Experimentalis et Applicata 8:49-64.

King, M. 1993. Species evolution. The role of chromosome change. Cambridge University Press, Cambridge, UK.

Kirkpatrick, M., and N. Barton. 1997. Evolution of species' range. American Naturalist 150:1-23.

Kohlmann, B., H. Nix, and D. D. Shaw. 1988. Environmental predictions and distributional limits of chromosomal taxa in the Australian grasshopper Caledia captiva (F.). Oecologia 75:483-93.

Lamb, R. J., and P. A. MacKay. 1987. Acyrthosiphon kondoi influences alata production by the pea aphid, A. pisum. Entomologia Experimentalis et Applicata 45:195-198.

Lui, R., and C. Keller. 1987. The effect of logistic growth on interacting populations that disperse to avoid crowding. Communications in Partial Differential Equations 12:11171132.

Lynch, J. D. 1989. The gauge of speciation: on the frequencies of modes of speciation. Pages 527-553 in D. Otte and J. A. Endler, editors. Speciation and its consequences. Sinauer, Sunderland, Massachusetts, USA.

MacArthur, R. H. 1972. Geographical ecology. Patterns in the distribution of species. Harper \& Row, New York, New York, USA.

MacLean, W., and R. Holt. 1979. Distributional patterns in St. Croix Sphaerodactylus lizards: the taxon cycle in action. Biotropica 11:189-185.

Mayr, E. 1963. Animal species and evolution. Harvard University Press, Cambridge, Massachusetts, USA.

McLaughlin, J. F., and J. Roughgarden. 1993. Species interactions in space. Pages 89-98 in R. E. Ricklefs and D. Schluter, editors. Species diversity in ecological communities. Historial and geographical perspectives. University of Chicago Press, Chicago, Illinois, USA.

Miller, R. S. 1967. Pattern and process in competition. Advances in Ecological Research 4:1-74.

Mimura, M., and K. Kawasaki. 1980. Spatial segregation in competitive interaction-diffusion equations. Journal of Mathematical Biology 9:49-64.

Murray, J. D. 1989. Mathematical biology. Springer-Verlag, New York, New York, USA.

NAG. 1999. Numerical Algorithms Group. <www.nag.co. uk> Oxford, UK.

Namba, T. 1989. Competition for space in a heterogeneous environment. Journal of Mathematical Biology 27:1-16.

Namba, T., and M. Mimura. 1980. Spatial distribution of competing populations. Journal of Theoretical Biology 87: 795-814.

Neet, C. R. and J. Hausser. 1990. Habitat selection in zones of parapatry contact between the common shrew Sorex araneus and Millet's shrew Sorex coronatus. Journal of Animal Ecology 59:235-250.

Nisbet, R. M., and W. S. C. Gurney. 1982. Modelling fluctuating populations. John Wiley \& Sons, New York, New York, USA.

Okubo, A. 1980. Diffusion and ecological problems: mathematical models. Springer-Verlag, New York, New York, USA.

Okubo, A., P. K. Maini, M. H. Williamson, and J. D. Murray. 1989. On the spatial spread of the grey squirrel in Britain. Proceedings of the Royal Society of London, Series B 238: 113-125.

Pianka, E. R. 1988. Evolutionary ecology. Harper \& Row, New York, New York, USA.

Pielou, E. C. 1974. Competition on an environmental gradient. Pages 185-204 in S. Levin, editor. Mathematical problems in biology. Lecture notes in biomathematics number 2. Springer-Verlag, New York, New York, USA.

Pulliam, H. R. 1996. Sources and sinks: empirical evidence and population consequences. Pages 45-69 in O. L. Rhodes, Jr., R. K. Chesser, and M. H. Smith, editors. Population dynamics in ecological space and time. University of Chicago Press, Chicago, Illinois, USA.

Ribeiro, J. M., and A. Spielman. 1986. The satyr effect: a model predicting parapatry and species extinction. American Naturalist 128:513-528.

Roughgarden, J. 1979. Theory of population genetics and 
evolutionary ecology: an introduction. MacMillan, New York, New York, USA.

Shigesada, N. 1984. Spatial distribution of rapidly dispersing animals in heterogeneous environments. Pages 478-491 in S. Levin and T. Hallam, editors. Mathematical ecology. Lectures notes in biomathematics number 54. Springer-Verlag, New York, New York, USA.

Shigesada, N., and K. Kawasaki. 1997. Biological invasions: theory and practice. Oxford University Press, Oxford, UK

Shigesada, N., K. Kawasaki, and E. Teramoto. 1979. Spatial segregation of interacting species. Journal of Theoretical Biology 79:83-99.

Sinclair, A. R. E. 1989. Population regulation in animals Pages 197-240 in J. M. Cherrett, editor. Ecological concepts. Blackwell, Oxford, UK.

States, J. B. 1976. Local adaptations in chipmunk (Eutamias amoenus) populations and evolutionary potential at species' borders. Ecological Monographs 46:221-256.

Steinberg, E. K., and P. Kareiva. 1997. Challenges and opportunities for empirical evaluation of "spatial theory."
Pages 318-332 in E. Tilman and P. Kareiva, editors. Spatial ecology. The role of space in population dynamics and interspecific interactions. Princeton University Press, Princeton, New Jersey, USA.

Terborgh, J. 1985. The role of ecotones in the distribution of Andean birds. Ecology 66:1237-1246.

Thaeler, C. 1974. Four contacts between ranges of different chromosome forms of the Thomomys talpoides complex (Rodentia: Geomyidae). Systematic Zoologist 23:343-354.

Turchin, P. 1998. Quantitative analysis of movement: measuring and modeling population redistribution in animals and plants. Sinauer, Sunderland, Massachusetts, USA.

White, M. J. D. 1973. Animal cytology and evolution. Cambridge University Press, Cambrigde, UK.

Wiens, J. A., and J. T. Rotenberry. 1981. Habitat associations and community structure of birds in shrub steppe environments. Ecological Monographs 51:21-42.

Witelski, T. P. 1997. Segregation and mixing in degenerate diffusion in population dynamics. Journal of Mathematical Biology 35:695-712. 